\section{Umweltplanung ante portas?}

\section{Es könnte ein ökologischer Knüller werden, was auf Seite 18 im Koalitions- vertrag zwischen SPD und Bündnis 90/ Die Grünen steht: „Die neue Bundes- regierung wird eine nationale Nachhaltigkeitsstrategie mit konkreten Zielen erarbeiten. Dies geschieht im Dialog mit den wichtigsten Gruppen. Die nationale Nachhaltigkeitsstrategie ist ein wichtiges Instrument zur Förderung ökologischer Innovationen wie auch zur Umsetzung der Agenda 21." Doch konkrete Schritte stehen bisher noch aus. Hierfür liegen verwertbare infernationale Erfahrungen vor.}

$\mathrm{N}$ Von Anneli Rüling und Axel Volkery ach einem halben Jahr Stillschweigen scheint es jetzt loszugehen: Ein sogenannter ,nationaler Rat für nachhaltige Entwicklung" wird bis 2002 eine Strategie ausarbeiten - dies hat zumindest Bundesumweltminister Trittin kürzlich angekündigt (1). Endlich steht auch im einstigen grünen Musterland eine strategische Neuausrichtung der Umweltpolitik auf der Agenda. Langfristige Orientierung statt kleiner Schritte im Takt der Legislaturperioden und Querschnitts- statt Ressortdenken scheinen sich damit endlich durchzusetzen. Die spannende Frage ist, was nun tatsächlich passieren wird. Eigentlich bedeutet eine „nationale Nachhaltigkeitsstrategie“, daß die ökologischen, ökonomischen und sozialen Zusammenhänge einer nachhaltigen Entwicklung definiert und in konkrete Ziele und Maßnahmen umgesetzt werden. Die Federführung müßte daher eigentlich beim Bundeskanzler liegen. Allerdings läßt der Koalitionsvertrag vermuten, daß Nachhaltigkeit weitgehend auf Umweltpolitik reduziert wird. Unter den gegebenen Vorzeichen ist also eher ein nationaler Umweltplan zu erwarten. Dies legen auch die Erfahrungen der europäischen Nachbarn nahe, die einen solchen bereits umgesetzt haben eine Nachhaltigkeitsstrategie, die alle drei Dimensionen umfaßt, gibt es noch nicht. Was aber ist von einem nationalen Umweltplan zu erwarten?

\section{Komplexe Umweltprobleme...}

Umweltpolitik steht vor einer Zäsur. Die ,alten“ Umweltprobleme sind weitgehend bewältigt worden. Die neuen Umweltprobleme haben aber eine völlig andere Dimension: Sie stehen in keinem direkten räumlichen und zeitlichen
Zusammenhang, sind teilweise nicht sicht- und meßbar und reduzieren schleichend die Umweltqualität. Probleme wie Klimaveränderung, Flächen- und Ressourcenverbrauch, Kontamination, Verlust der Artenvielfalt und hohe Ozonbelastung lassen sich über Ordnungsrecht und End-of-Pipe-Technologien allein nicht angehen. Komplexe Umweltprobleme erfordern komplexe Lösungen.

\section{...und komplexe Lösungsansätze}

Umweltplanung scheint - zumindest der Theorie nach - ein solcher „Königsweg“ der Umweltpolitik zu sein. Dieser Ansatz verspricht einen gesellschaftlichen Handlungsrahmen zur integrierten Problembearbeitung und gemeinsamen Suche nach wirtschafts- und sozialverträglichen Lösungen: In einem offenen Diskurs müßten die gesellschaftlichen Akteure langfristige Umweltziele formulieren, die durch kurzfristige Ziele handhabbar gemacht werden. Im Gegenzug gewinnen die Akteure eine langfristige Planungsund Investitionssicherheit sowie verläßliche Rahmenbedingungen für ökologische Forschung und Innovationen.

Tatsächlich wird in deutschen Fachkreisen schon seit einigen Jahren über Umweltplanung debattiert, zu nennen sind hier insbesondere das 1998 vom Bundesministerium für Umwelt, Naturschutz und Reaktorsicherheit nach einen Konsultationsprozeß vorgestellte umweltpolitische Schwerpunktprogramm und die Vorarbeiten der Enquete-Kommission „Schutz des Menschen und der Umwelt", aber auch die Stellungnahmen des Sachverständigenrates für Umweltfragen und die Nachhaltigkeitsstudie des Umweltbundesamtes. Die Vorarbeiten sind also geleistet. Allerdings fehlte der Regierung Kohl der politische Umsetzungswille.
In vielen EU-Ländern existieren bereits Umweltpläne in unterschiedlichen Ausprägungen. Die Erfahrungen zeigen, daß damit die umweltpolitischen Kapazitäten erweitert und strategisch neu ausgerichtet werden konnten. Eine vergleichende Studie über die Niederlande, Österreich und Schweden macht deutlich, daß folgende Kriterien bei der Erstellung eines nationalen Umweltplans beachtet werden sollten (2):

\section{Drei Gütekriterien}

\section{Starke Zielstruktur}

Die Umweltziele sollten möglichst quantifiziert und mit einem klaren Zeithorizont versehen sein. Zur Operationalisierung sind meßbare kurz- und mittelfristige Zwischenziele wichtig. Im weiteren Planungsprozeß ist ein Monitoring der Zielerreichung und die Fortschreibung bzw. Weiterentwicklung der Ziele notwendig. Die Niederlande bieten dazu exzellente Beispiele. Dort wurden aus Reduktionsanforderungen der nächsten 20 Jahre konkrete Reduktionsziele für kurze Zeitspannen errechnet und werden weiterentwickelt.

\section{Vollständigkeit}

Für eine integrierte und präventive Umweltpolitik muß neben „klassischen“ Themen wie Emissionen auch die „Inputseite“ betrachtet werden. Energie-, Material- und Flächenverbrauch sollten daher unbedingt behandelt werden. Österreich hat diese Themen bearbeitet und teilweise konkrete Ziele entwickelt - beispielsweise die langfristige Reduktion des Stoffdurchsatzes um 90 Prozent. Leider wird dieser Plan momentan nicht aktiv weiter verfolgt. Der gerade vorgelegte schwedische Umweltplan enthält ebenfalls ambitionierte Ziele für die Senkung des Materialverbrauchs und die Erhöhung der Ressourcenproduktivität.

\section{Klare Verantwortlichkeiten}

Mit den für Umweltprobleme verantwortlichen Akteuren sollten konkrete Ziele verbindlich vereinbart werden. Durch wirkungsvolle Sanktionsmechanismen muß sichergestellt werden, daß diese Abkommen auch erfüllt werden. Hier sind wiederum die Niederlande beispielhaft. Gemeinsam mit den wichtigen Verursacherbranchen setzen die Behörden Reduktionspflichten und -fristen fest, die dann in Covenants, einer bindenden Absichtserklärung, festgeschrieben werden.

Essentiell für eine erfolgreiche gesellschaftliche Verankerung des Umweltplans ist, wie der Vergleich zeigt, die organisatorische und politische Gestaltung des Planungsprozesses (3). 
Die Planung erfordert vor allem ausreichend Zeit: In Österreich dauerte die Planerstellung fünf, in den Niederlande sechs Jahre. Gefordert ist also ein langer Atem und vor allem die Lernund Kompromißfähigkeit aller Beteiligten. Initiator des Planungsprozesses muß die Regierung sein. Sie sollte die Akteure einberufen, den Prozeß moderieren und gleichzeitig das Allgemeinwohl vertreten.

Politisches Gewicht erhält die Umweltplanung vor allem durch eine starke Institutionalisierung. Dies bedeutet, daß Nachhaltigkeit als Querschnittsthema in alle Ressorts integriert werden muß. Befördert werden kann dies beispielsweise durch

- einen ,Nachhaltigkeitsrat“, in dem alle relevanten Minister vertreten sind (wie beispielsweise in Schweden),

durch einen „Nachhaltigkeitsbeauftragten“ in allen Ministerien (wie die ,green ministers“ in Großbritannien) oder

- ein Vetorecht des Umweltministers.

Ferner bedarf es einer zentralen Steuerungsinstanz, in der Beauftragte aus Ministerien, Bundestag, Bundesländer, Wissenschaft und den Interessenorganisationen vertreten sind.

\section{Gesellschaftlicher Lernprozeß}

Für die Akzeptanz und den Erfolg der Strategie ist eine möglichst pluralistische Interesseneinbindung bei der Planerstellung vonnöten - alle wichtigen gesellschaftlichen Gruppen (Parteien, Verbände, Gewerkschaften, Kirchen, Umweltverbände, Kommunen etc.) sollten frühzeitig am Planungsprozeß beteiligt werden, mit klaren Rechten und Pflichten. Ferner muß gesetzlich abgesichert werden, daß die Planung fortgeschrieben wird. In den Niederlanden ist beispielsweise festgelegt, daß alle vier Jahre ein neuer nationaler Umweltplan erstellt und alle zwei Jahre durch Umweltzustandsberichte evaluiert wird.

Schließlich muß die breite Öffentlichkeit - etwa über die Medien, Schulen oder Umweltinitiativen - in die Umsetzung des Umweltplans eingebunden werden. Denn zu den wichtigsten Akteuren zählen die Verbraucher, vor allem in Bereichen, die Verhaltensänderungen erfordern, wie insbesondere der Verkehr. Für diesen Sektor gibt es bisher in keinem Land zufriedenstellende Lösungen.

Die Beteiligung der verschiedenen gesellschaftlichen Gruppen im Planungsprozeß erweitert die politischen Handlungsspielräume. Die Akteure stellen die theoretische und praktische Kompe- tenz für den notwendigen gesellschaftlichen Wandel bereit. Indem die relevanten Gruppen von Anfang an ,mit ins Boot geholt werden“, soll späteren Protesten und Konflikten vorgebeugt werden. Damit wird eine Kapazitätserweiterung und eine neuartige Legitimation langfristiger angelegter Politik möglich.

Ein solcher gesellschaftlicher Lernprozeß wird und kann nicht idealiter vonstatten gehen. Erfolge sind aber dennoch möglich, wie die internationalen Erfahrungen zeigen. Umweltplanung bietet die Chance, die Gewinner einer ökologischen Modernisierung zusammenzuführen und gegenüber traditionellen Bremsern zu stärken. Entscheidend ist hierfür letztlich der politische Wille der Akteure.

Soweit das Konzept. Wie aber geht es realpolitisch weiter? Spannend bleibt, wie ein nationaler Rat für nachhaltige Entwicklung institutionalisiert wird und mit welcher Beteiligung gesellschaftlicher Gruppen Umweltziele erarbeitet werden. Interesse bekundet hat beispielsweise der Deutsche Gewerkschaftsbund, der die Erarbeitung einer nationalen Nachhaltigkeitsstrategie begrüßt. Er forderte kürzlich, im Bündnis für Arbeit langfristige Rahmenbedingungen und konkrete Umweltziele festzulegen; hierzu gab es in der Folge auch im Arbeitgeberlager eine positive Resonanz (4). Bleibt zu hoffen, daß aus diesen zaghaften Ansätzen wirkliche Politik wird. Die rot-grüne Regierung hat sich selbst einen klaren Handlungsauftrag gegeben.

\section{Anmerkungen}

(1) „Ein Verlierer startet durch", Frankfurter Rundschau vom 19.5.1999.

(2) Rüling, Anneli/ Axel Volkery: Umweltplanung nach Rio: Niederlande, Österreich und Schweden im Vergleich, FFUReport 99-1, Forschungsstelle für Umweltpolitiik, Berlin. (3) Jänicke, Martin et al.: Nationale Umweltpläne in ausgewählten Industrieländern. Berlin, Heidelberg 1997. Vgl. auch Ökologisches Wirtschaften 2/97, S. 6-7.

(4) Frankfurter Rundschau vom 29.4.1999; DGB-Positionspapier zum Themen- und Fachdialog „Umwelt” im Bündnis für Arbeit: Arbeit und Umwelt, 28.4.1999.

\section{Die Autorlnnen}

Anneli Rüling und Axel Volkery studieren politische Wissenschaft an der FU Berlin.

Kontakt: A. Rüling, Kastanienallee 55,

10119 Berlin, Tel. 030/ 4499703,

E-mail: rueling@medea.wz-berlin.de;

A. Volkery, Hindenburgdamm 91, 12203 Berlin,

Tel./ Fax 030/ 8342629, E-mail: volkery@aol.com

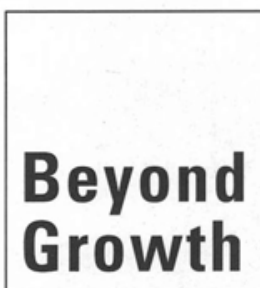

Heinrich Böll Stiftung in Kooperation mit Wuppertal Institut VÖÖ/ESEE/ISEE

\section{Nachhaltige Entwicklung versus \\ Wirtschaftswachstum?}

Internationale Konferenz

24.-26. 9. 1999 in Berlin

Themen u.a.

- Grenzen der Ökoeffizenz

- Gutes Leben durch

Wirtschaftswachstum?

- Globalisierung, Wachstum und Nachhaltigkeit

- Politik und Wachstum -

Vom Zwang zur Wahl?

Mit Herman Daly, Thilo Bode, Manfred Max-Neef, Sabine O'Hara, Reinhard Loske, Ernst Ulrich von Weizsäcker u. a.

Info: Fon 030-28534-243, Fax -108 www.wupperinst.org/Beyond_Growth

\section{Beilagenhinweis}

\section{Unsere Publikation enthält eine Beilage von der Umweltbank.}

\section{Wir bitten um freundliche Beachtung.}

Einen Überblick über alle
bisher erschienen Hefte des
Informationsdienstes
"Ökologisches Wirtschaften"
finden Sie auf unseren Internetseiten
unter der Adresse:
http://www.oekom.de
Wir freuen uns auf Ihren Besuch!


(c) 20I0 Authors; licensee IÖW and oekom verlag. This is an article distributed under the terms of the Creative Commons Attribution Non-Commercial No Derivates License (http://creativecommons.org/licenses/by-nc-nd/3.o/), which permits unrestricted use, distribution, and reproduction in any medium, provided the original work is properly cited. 